Pesq. Vet. Bras. 38(5):1005-1013, maio 2018

\title{
Aspectos ultrassonográficos, biometria e dopplerfluxometria ocular do bugio ruivo (Alouatta fusca) ${ }^{1}$
}

\author{
Lívia P. Souza ${ }^{2 *}$, Natalie B. Merlini ${ }^{3}$, Zara Bortolini ${ }^{4}$, Thiago R. Muller ${ }^{5}$, \\ Carlos R. Teixeira ${ }^{6}$, Marilia G. Luciani ${ }^{7}$, Daiane S. Souza ${ }^{8}$ e Luiz C. Vulcano ${ }^{9}$
}

\begin{abstract}
Souza L.P., Merlini N.B., Bortolini Z., Muller T.R., Teixeira C.R., Luciani M.G., Souza D.S. \& Vulcano L.C. 2018. [Ultrasonography aspects, biometry and Doppler of howler monkey (Alouatta fusca) ocular bulb]. Aspectos ultrassonográficos, biometria e dopplerfluxometria ocular do bugio ruivo (Alouatta fusca). Pesquisa Veterinária Brasileira 38(5):1005-1013. Centro de Ciências Agroveterinárias, Universidade do Estado de Santa Catarina, Avenida Luiz de Camões 2090, Conta Dinheiro, Lages, SC 88520-000, Brazil. E-mail: li.pasini.vet@gmail.com

This study aimed to describe the sonographic features of normal ocular structures, the ocular biometry and Doppler parameters of the internal ophthalmic artery and central retinal artery of the Alouatta fusca. Twenty ocular ultrasonographic examinations were perform in ten primate species of the Alouatta fusca. Proceeded to the sonographic description of the ocular structures and later biometrics was obtained in four distances: (D1) corresponding to the anterior chamber (D2) lens thickness (D3) vitreous chamber and (D4) axial length of the eyeball. Doppler ultrasound evaluated internal ophthalmic artery and central retinal artery as for the resistivity Index (RI), pulsatility index (PI), peak systolic velocity (PSV) and end diastolic velocity (EDV). The values of biometrics and flowmetry underwent comparison test between genders and sonographic views, by Student $t$ test. The anatomical sonographic description of the ocular structures and vasculature of the red howler were similar to species such as dog, cat and man. The biometric average values found were $2.1 \pm 0.38 \mathrm{~mm}$ for $\mathrm{D} 1,3.7 \pm 0.30 \mathrm{~mm}$ for $\mathrm{D} 2,10.4 \pm 0.78 \mathrm{~mm}$ for D3 and $19.3 \pm 1.64 \mathrm{~mm}$ for D4. The Doppler values of the ophthalmic artery and central retinal artery found were: PSV of $25.6 \mathrm{~cm} / \mathrm{s}$ and $14.6 \mathrm{~cm} / \mathrm{s} ; \operatorname{VDF} 15.8 \mathrm{~cm} / \mathrm{s}$ and $10.7 \mathrm{~cm} / \mathrm{s}$; IR 0.7 and 0.5 ; IP 1.4 and 0.8 .
\end{abstract}

INDEX TERMS: Ultrasonography, biometry, Doppler, howler monkey, Alouatta fusca, ocular bulb ophthalmology, ultrasound.

\footnotetext{
${ }^{1}$ Recebido em 28 de março de 2017.

Aceito para publicação em 23 de maio de 2017.

2 Doutoranda, Centro de Ciências Agroveterinárias (CAV), Universidade do Estado de Santa Catarina (UDESC), Avenida Luiz de Camões 2090, Conta Dinheiro, Lages, SC 88520-000, Brasil. *Autor para correspondência: li.pasini.vet@gmail.com

${ }^{3}$ Doutoranda, Faculdade de Medicina Veterinária e Zootecnia (FMVZ), Universidade Estadual Paulista (Unesp), Campus de Botucatu, Rua Prof. Dr. Walter Mauricio Correra s/n, Distrito de Rubião Junior, Botucatu, SP 18618-970, Brasil. E-mail: natalie_merlini@hotmail.com

${ }^{4}$ Doutoranda, Faculdade de Medicina Veterinária e Zootecnia (FMVZ), Universidade Estadual Paulista (Unesp), Campus de Botucatu, Rua Prof. Dr. Walter Mauricio Correra s/n, Distrito de Rubião Junior, Botucatu, SP 18618-970. E-mail: zarabortolini@yahoo.com.br

${ }^{5}$ Professor Doutor, Centro de Ciências Agroveterinárias (CAV), Universidade do Estado de Santa Catarina (UDESC), Avenida Luiz de Camões 2090, Conta Dinheiro, Lages, SC 88520-000. E-mail: mullerusa@hotmail.com
}

\footnotetext{
${ }^{6}$ Docente, Faculdade de Medicina Veterinária e Zootecnia (FMVZ), Universidade Estadual Paulista (Unesp), Campus de Botucatu, Rua Prof. Dr. Walter Mauricio Correra s/n, Distrito de Rubião Junior, Botucatu, SP 18618-970. E-mail: teixeiracr@fmvz.unesp.br

${ }^{7}$ Residente no Setor de Diagnóstico por Imagem, Centro de Ciências Agroveterinárias (CAV), Universidade do Estado de Santa Catarina (UDESC), Avenida Luiz de Camões 2090, Conta Dinheiro, Lages, SC 88520-000. E-mail: marilia_luciani@hotmail.com

${ }^{8}$ Residente no Setor de Diagnóstico por Imagem, Centro de Ciências Agroveterinárias (CAV), Universidade do Estado de Santa Catarina (UDESC), Avenida Luiz de Camões 2090, Conta Dinheiro, Lages, SC 88520-000. E-mail: mvsturmer@gmail.com

${ }^{9}$ Docente, Faculdade de Medicina Veterinária e Zootecnia (FMVZ), Universidade Estadual Paulista (Unesp), Campus de Botucatu, Rua Prof. Dr. Walter Mauricio Correra s/n, Distrito de Rubião Junior, Botucatu, SP 18618-970. E-mail: vulcano@fmvz.unesp.br
} 
RESUMO.- Este estudo teve como objetivo geral descrever os aspectos ultrassonográficos normais das estruturas do bulbo ocular de primatas da espécie Alouatta fusca, determinando ainda os valores da biometria ocular, e os parâmetros da dopplerfluxometria ocular das artérias oftálmica interna e central da retina. Vinte exames ultrassonográficos oculares foram realizados em dez primatas da espécie Alouatta fusca clinicamente saudáveis, sem sinais de doença ocular ao exame oftalmológico. Procedeu-se a descrição ultrassonográfica das estruturas oculares e posteriormente a biometria foi obtida em quatro distâncias distintas: (D1) correspondentes à câmara anterior, (D2) espessura da lente, (D3) profundidade da câmara vítrea e (D4) comprimento axial do bulbo ocular. $\mathrm{Na}$ dopplerfluxometria foram avaliadas as artérias oftálmica interna e central da retina quanto ao índice de resistividade (IR), de pulsatilidade (IP), e as velocidades do pico sistólico (VPS) ediastólica final (VDF). Os valores de biometria foram submetidos a teste de comparação quanto ao gênero dos animais e cortes ultrassonográficos, utilizando o teste t de Student. 0 mesmo teste foi realizado para comparação dos resultados de dopplerfluxometria entre fêmeas e machos. A descrição ultrassonográfica das estruturas oculares e vascularização do bugio ruivo mostraram-se semelhantes a espécies como o cão, o gato e o homem. Os valores biométricos médios encontrados foram de 2,1 $\pm 0,38 \mathrm{~mm}$ para $\mathrm{D} 1,3,7 \pm 0,30 \mathrm{~mm}$ para D2, $10,4 \pm 0,78 \mathrm{~mm}$ para D3 e $19,3 \pm 1,64 \mathrm{~mm}$ para D4. Os valores de dopplerfluxometria da artéria oftálmica e da artéria central da retina foram respectivamente: VPS de $25,6 \mathrm{~cm} / \mathrm{s}$ e $14,6 \mathrm{~cm} / \mathrm{s} ;$ VDF de $15,8 \mathrm{~cm} / \mathrm{s}$ e $10,7 \mathrm{~cm} / \mathrm{s}$; IR de 0,7 e 0,$5 ;$ IP de 1,4 e 0,8 .

TERMOS DE INDEXAÇÃO: Ultrassonografia, biometria, dopplerfluxometria, ocular, bugio ruivo, Alouatta fusca, oftalmologia, ecografia, Doppler.

\section{INTRODUÇÃO}

Os primatas não humanos mantidos em cativeiro têm grande importância para a educação e pesquisa (Montiani-Ferreira et al. 2008). 0 estudo anatômico destas espécies, crescente nos últimos 30 anos, destina-se à obtenção de dados acerca de sua constituição morfológica, bem como de conseguir subsídios para comparação entre espécies semelhantes anatômica, fisiológica e etologicamente, como o ser humano (Auricchio 1995, Marques 2005).

O sistema ocular dos primatas não humanos é basicamente semelhante ao dos animais domésticos, constituído pelo bulbo ocular propriamente dito e seus anexos (Mattoon \& Nyland 2005, Samuelson 2007, Pippi \& Gonçalves 2009).

A irrigação do bulbo ocular é complexa (Dyce et al. 2004). Na maioria dos animais domésticos, o maior suprimento sanguíneo da órbita provém de pequenos vasos arteriais originados da artéria oftálmica externa, a qual é uma ramificação da artéria maxilar interna, originada da artéria carótida externa (Diniz et al. 2004, Carvalho et al. 2009, Pippi \& Gonçalves 2009). Contudo, em primatas não humanos, a microcirculação total do bulbo ocular e a maior parte da circulação orbital é suprida pela artéria oftálmica interna sendo esta, uma ramificação emitida pela artéria carótida interna (Diniz et al. 2004).

Na órbita, a artéria oftálmica interna está localizada entre o músculo reto lateral e o nervo óptico. Em uma localização mais cranial ela cruza o nervo óptico em sentido medial e dá origem à grande parte de seus ramos, sendo a artéria central da retina, artérias ciliares posteriores, artéria lacrimal e artérias supraorbitais (Diniz et al. 2004).

Assim como descrita em cães e gatos, a drenagem venosa é realizada por veias oftálmicas que penetram no bulbo ocular sob a forma de veias ciliares posteriores, que dão origem às veias da retina - o plexo aquoso angular, que tem como unidade venosa as veias coletoras e as veias ciliares anteriores (Pippi \& Gonçalves 2009).

O olho é considerado um órgão ideal para o exame ultrassonográfico por ser de fácil acesso e conter várias superfícies ou interfaces reflexivas (Morgan 1989). Este método de diagnóstico tem sido uma ferramenta essencial no exame oftalmológico por delinear e caracterizar tecidos moles do olho e da órbita (Gonzalez et al. 2001, Hernández-Guerra et al. 2007), sendo uma técnica minimamente invasiva, acessível e que proporciona ao clínico veterinário informações relevantes a fim de auxiliar no diagnóstico, tratamento e prognóstico de diversas afecções (Mattoon \& Nyland 2005).

As indicações para exame ultrassonográfico do bulbo ocular incluem trauma, exoftalmia evidente, detecção e evolução de corpos estranhos ou massas intraoculares ou orbitais, luxação da lente, glaucoma, uveíte, descolamento de retina (diferenciando sua origem regmatogênica, exsudativa ou por tração) e, principalmente, quando houver opacificação ocular média, completa ou parcial, a fim de se determinar a ocorrência de hemorragia, catarata, edemacorneal, exsudação fibrinosa, hifema ou hipópio (Gonzalez et al. 2001, Hernández-Guerra et al. 2007).

A biometria ocular consiste na mensuração do bulbo ocular e de suas estruturas internas, como a câmara anterior, a lente e a câmara vítrea (Gonzalez et al. 2001, Hernández-Guerra et al. 2007, Toni et al. 2010). Tais mensurações são importantes para a avaliação e identificação de alterações, bem como no auxílio à determinação do tamanho da lente intraocular em casos de implantes e próteses de globo ocular pós-enucleação (Gonzalez et al. 2001, Toni et al. 2010).

A ultrassonografia é utilizada desde 1956 para o diagnóstico de doenças oculares em seres humanos. Na veterinária, este método foi descrito pela primeira vez em 1968, com a aplicação da ultrassonografia unidimensional para análise de bulbos oculares de animais portadores de opacificação de meios transparentes. Já a utilização da ultrassonografia modo-B no diagnóstico de doenças oculares na veterinária foi descrita em 1980 (Mattoon \& Nyland 2005).

A ultrassonografia em modo B concede a formação de uma imagem bidimensional em tempo real, numa escala de cinza, permitindo a identificação de estruturas, sua localização, formato, aspecto e contorno. Já o modo A é basicamente utilizado para mensurações biométricas e detecção de mobilidade dos tecidos, conseguida através da conversão da velocidade do som no respectivo tecido (tempo) em distância (Hernández-Guerra et al. 2007).

0 recurso chamado Color Doppler Pulsado tem sido de grande contribuição para o estudo ultrassonográfico do bulbo ocular. Tal exame permite a identificação de alterações vasculares de maneira rápida e não invasiva, auxiliando o oftalmologista no diagnóstico de afecções como o glaucoma, a retinopatia diabética, processos inflamatórios e neoplásicos e doenças vasculares sistêmicas (como a hipertensão), 
permitindo estabelecer uma conduta terapêutica adequada (Diniz et al. 2004, Hernández-Guerra et al. 2007).

Este estudo teve como objetivo geral descrever os aspectos ultrassonográficos normais das estruturas do bulbo ocular do bugio ruivo (Alouatta fusca), bem como relatar a biometria ocular e os parâmetros da dopplerfluxometria das artérias oftálmica interna e central da retina, a fim de aumentar as bases de dados para estudos científicos futuros e auxiliar na rotina clínica desta e de outras espécies.

\section{MATERIAL E MÉTODOS}

0 estudo foi aprovado pelo comitê de ética da FMVZ/UNESP, Botucatu, com o número de protocolo 126/2012. Vinte bulbos oculares de dez primatas da espécie Alouatta fusca foram avaliados, sendo cinco machos e cinco fêmeas, com peso e idade variando de $3 \mathrm{~kg}$ a $6 \mathrm{~kg}$ e 3 a 12 anos, respectivamente. Os animais foram provenientes do Centro de Medicina e Pesquisa em Animais Silvestres (CEMPAS), da Faculdade de Medicina Veterinária e Zootecnia da Unesp, Campus de Botucatu.

Após contenção com auxílio de puçá, os animais foram anestesiadosusando a associação de cloridrato de cetamina na dose de $7 \mathrm{mg} / \mathrm{kg}$ (Cetamin ${ }^{\circledR} 10 \%$, Syntec do Brasil), midazolan na dose de $5 \mathrm{mg} / \mathrm{kg}$ (Dormire ${ }^{\circledR} 0,5 \%$, Cristália Produtos Químicos e Farmacêuticos Ltda) e xilazina na dose de $0,3 \mathrm{mg} / \mathrm{kg}$ (Xilazin ${ }^{\circledR} 2 \%$, Syntec do Brasil) via intramuscular. Foram incluídos somente animais hígidos no estudo, para isso, antes de iniciar o exame ultrassonográfico e após anestesia, realizou-se o exame físico geralpelo veterinário do Serviço de Clínica do Centro de Medicina e Pesquisa em Animais Silvestres (FMVZ-Unesp), e exame oftálmico por um veterinário especializado do Serviço de Oftalmologia da FMVZ-Unesp.

A avaliação física geral abordou auscultação cardíaca e pulmonar, avaliação de mucosas e pulso, palpação de linfonodos e turgor cutâneo, já a avaliação oftálmica de rotina incluiu análise dos anexos oftálmicos, segmento anterior e posterior, realizada com auxílio de lâmpada de fenda portátil (SL-15 Kowa, Japan) e oftalmoscópio direto (WelchAllyn ${ }^{\circledR}$ ), sendo excluído do estudo qualquer bulbo ocular que apresentasse anormalidade.

Os exames ultrassonográficos foram realizados pelo mesmo ultrassonografista, que possuía experiência na área. Ao iniciar foi instilada uma gota de cloridrato de proximetacaína (Anesalcon ${ }^{\circledR} 0,5 \%$ - Alcon Laboratórios do Brasil) na córnea de cada bulbo ocular, para posterior utilização de gel estéril aquoso (SterileAquasonic ${ }^{\circledR}$ ) entre a córnea e o transdutor. Todos os animais foram posicionados em decúbito esternal, sendo necessário o auxilio de um ajudante para apoiar a cabeça e manter as pálpebras abertas, a fim de realizar o exame ultrassonográfico transcorneal.

Utilizou-se o aparelho ultrassonográfico Esaote MyLab 30 Vet Gold ${ }^{\circledast}$, dotado do recurso Doppler, utilizando-se os transdutores lineares multifrequenciais de 7,5 a $10 \mathrm{MHz}$ para a dopplerfluxometria e 18 a $20 \mathrm{MHz}$ para a avaliação da anatomia ultrassonográfica e biometria ocular. Para avaliação morfológica e biometria foi realizada a varredura completa do bulbo ocular em modo B e obtidas imagens em planos sagital e dorsal das estruturas, incluindo a córnea, a câmara anterior, a lente, a câmara vítrea, a parede posterior e o espaço retrobulbar.

Para a biometria, foram obtidas mensurações em milímetros da distância um (D1), entre o polo central posterior da imagem da córnea até a cápsula anterior da lente, correspondendo à profundidade da câmara anterior; distância dois (D2), entre a cápsula anterior da lente e a cápsula posterior da lente, equivalendo à espessura da lente; distância três (D3), distância da cápsula posterior da lente até a retina, compatível com a profundidade da câmara vítrea; e distância quatro (D4), obtida da face anterior da córnea até a retina, representando o comprimento axial total do bulbo ocular (Fig.1).
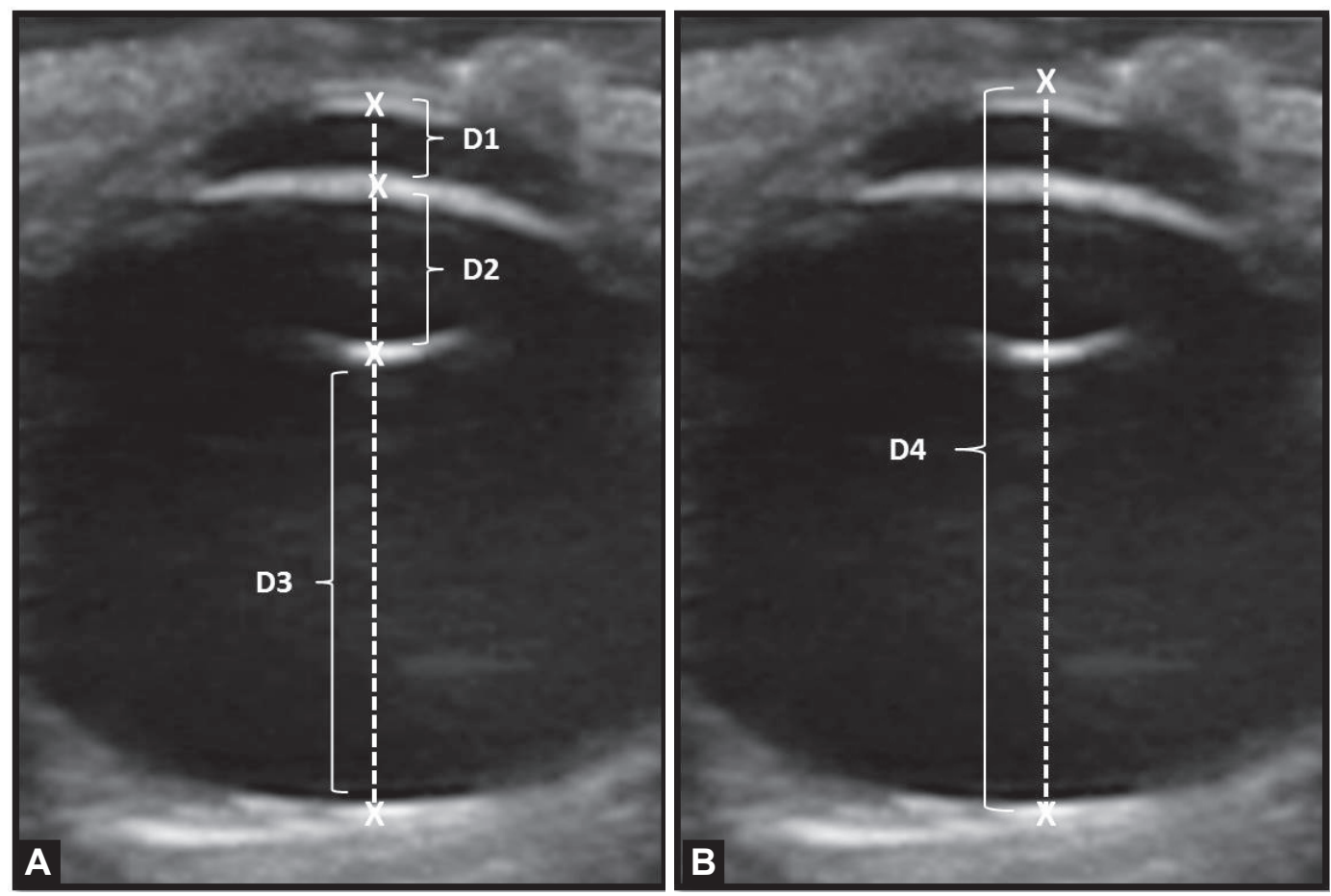

Fig.1. Imagem ultrassonográfica do bulbo ocular de um bugio ruivo (Alouatta fusca) obtida no plano sagital. Estão delimitadas pelas linhas brancas pontilhadas as distâncias obtidas na biometria, sendo representadas em (A) as distâncias D1 (profundidade da câmara anterior), D2 (espessura da lente) e D3 (profundidade da câmara vítrea) e em (B) a distância D4 (comprimento axial do bulbo). 
No estudo de dopplerfluxometria foram avaliadas as artérias oftálmica interna e artéria central da retina no plano dorsal. Primariamente avaliou-se o espaço retrobulbar e, posteriormente, caracterizou-se o fluxo pelo recurso Doppler. 0 cursor foi posicionado paralelo ao vaso de interesse para aquisição do traçado espectral. O fluxo da artéria oftálmica interna foi caracterizado em uma posição lateral e ventral quando profunda e medialmente ao nervo óptico em sua porção superficial. 0 registro do fluxo da artéria central da retina foi identificado no interior dos $5 \mathrm{~mm}$ próximos ao ponto central da parede posterior do bulbo ocular, praticamente sobreposta à imagem do nervo óptico.

A partir da mensuração de três ondas espectrais consecutivas de cada traçado (Fig.2), foram obtidos os valores de dopplerfluxometria calculados diretamente pelo aparelho ultrassonográfico. Os parâmetros hemodinâmicos avaliados foram o índice de resistividade (IR), índice de pulsatilidade (IP), velocidade do pico sistólico (VPS) e velocidade diastólica final (VDF).

Em todos os estudos debiometria e fluxometria, três mensurações de cada bulbo ocular foram colhidas, para obtenção de parâmetros mais fidedignos. As informações observadas em tais estudos foram compiladas em tabelas para obtenção de uma média e desvio padrão e, posteriormente, submetidas a testes de comparação pelo teste t de Student.
Para estudo comparativo entre gêneros, foram criados os grupos machos (GM) e fêmeas (GF), a fim de se verificar a existência de diferenças significativas entre as amostras em relação a biometria e a fluxometria. Quanto à biometria, os planos de corte anatômicos sagital (PS) e dorsal (PD) foram também comparados. Este teste foi feito para o bulbo ocular esquerdo e direito, sendo o nível de significância (p), também conhecido como t- crítico bilateral, estabelecido em 0,05.

\section{RESULTADOS}

Um bulbo ocular foi retirado do estudo de descrição anatômica e biometria devido à presença de uma sinéquia em porção lateral de córnea, detectada durante o exame oftalmológico em um bugio macho. Os valores da dopplerfluxometria da artéria oftálmica interna foram obtidos de um total de 20 bulbos oculares; já os da artéria central da retina foram obtidos de 18 bulbos oculares, devido à dificuldade de caracterização da onda espectral em dois animais.

À ecografia, o bulbo ocular apresentou-se, basicamente, como uma discreta estrutura arredondada bem delimitada por uma linha hiperecoica regular, preenchida por conteúdo anecoico em ambos os planos ultrassonográficos (Fig.3).
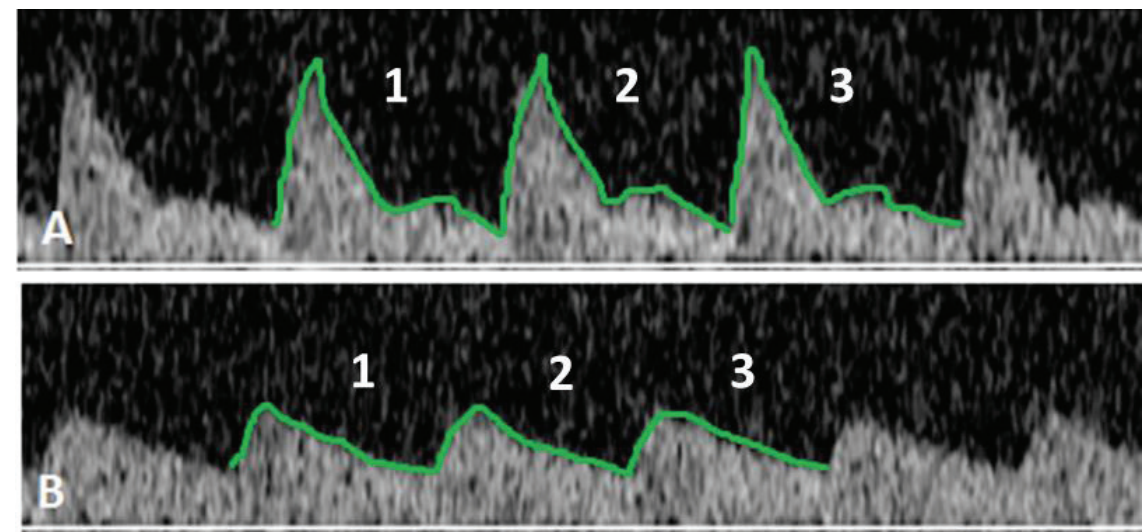

Fig.2. (A) Três mensurações obtidas no traçado espectral da artéria oftálmica interna do bulbo ocular de um bugio ruivo (Alouatta fusca).

(B) Três mensurações obtidas no traçado espectral da artéria central da retina.
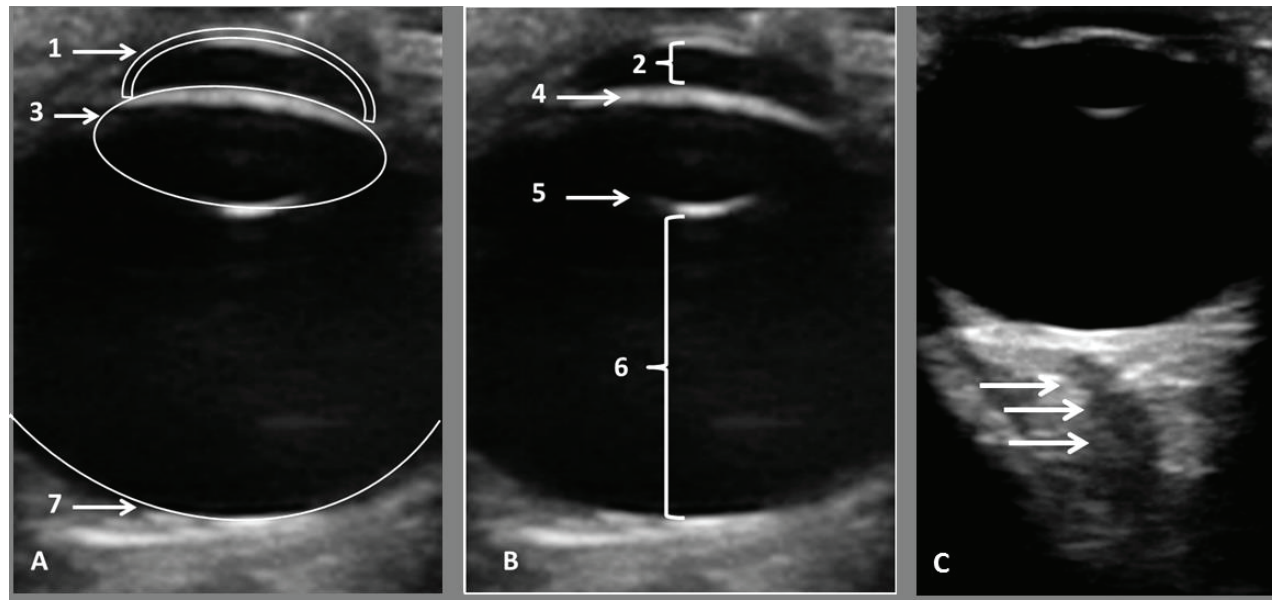

Fig.3. Imagens ultrassonográficas do bulbo ocular e espaço retrobulbar obtidas no plano sagital de um bugio ruivo (Alouatta fusca).

(A,B) Indicam as seguintes estruturas: córnea (1), câmara anterior (2), lente (3), cápsula lenticular anterior ou cápsula anterior da lente (4), cápsula lenticular posterior ou cápsula posterior da lente (5), câmara vítrea (6), parede posterior do bulbo ocular (7). (C) As setas brancas delimitam a região hipoecóica que representa o nervo óptico envolto pela gordura retrobulbar moderadamente ecogênica. 
A estrutura identificada mais próxima ao transdutor é a córnea, representada por uma fina linha hiperecoica semicircular. Posteriormente, observou-se pequeno espaço preenchido por conteúdo anecoico, a câmara anterior, tendo como delimitação caudal a cápsula anterior da lente, observada como uma linha hiperecoica lisa convexa.

A lente foi caracterizada por uma linha hiperecoica curvilínea convexa e côncava correspondendo, respectivamente, à cápsula lenticular anterior e posterior, preenchida por conteúdo anecoico. Em muitos casos, foi difícil a identificação de toda a cápsula lenticular em uma única imagem: a área central da superfície da cápsula anterior e posterior era visibilizada, enquanto suas laterais não eram observadas no mesmo corte, necessitando de varredura em leque para avaliação dos corpos ciliares laterais. Posterior à lente, observou-se a câmara vítrea como uma área completamente anecoica, delimitada em sua porção inicial pela cápsula posterior da lente e, em sua porção final, pela parede posterior do bulbo ocular. Em alguns cortes, linhas ecogênicas paralelas eram visibilizadas no conteúdo vítreo anecoico.

A parede posterior do bulbo ocular foi identificada como uma fina camada hiperecoica lisa e curvilínea caudal à câmara vítrea, representando o complexo retina-coróide-esclera, que não são individualizados ultrassonograficamente. Logo após a parede posterior bulbar visibiliza-se o espaço retrobulbar, área caracterizada por moderada ecogenicidade em função da gordura ali acumulada. Centralmente a essa porção, o nervo óptico é representado como uma estrutura hipoecóica em formato de funil. Todas as estruturas descritas são indicadas na Figura 3.
Os resultados médios da biometria ocular encontrados dentre as três mensurações realizadas para as diferentes regiões avaliadas foram (Quadro 1): 2,1 $\pm 0,38 \mathrm{~mm}$ para a mensuração da câmara anterior (D1) do olho esquerdo e 2,1 $\pm 0,35 \mathrm{~mm}$ para o bulbo direito; $3,7 \pm 0,23 \mathrm{~mm}$ para a espessura da lente (D2) do bulbo ocular esquerdo e $3,7 \pm 0,30 \mathrm{~mm}$ para o direito; $10,4 \pm 0,78 \mathrm{~mm}$ para a profundidade da câmara vítrea (D3) do bulbo esquerdo e $10,3 \pm 0,74 \mathrm{~mm}$ para o direito; $19,3 \pm 1,64 \mathrm{~mm}$ para o comprimento axial do bulbo ocular (D4) esquerdo e $19,3 \pm 1,33 \mathrm{~mm}$ para o direito. Não houve diferença significativa entre ambos os olhos, direito e esquerdo.

Testes comparativos não demonstraram diferença significativa entre os grupos machos e fêmeas (Quadros 2 e 3), nem mesmo entre os planos de corte sagital e dorsal (Quadro 4) para os parâmetros de biometria, com valores de t crítico bilateral maiores que o nível de significância $(0,05)$.

0 traçado espectral da artéria oftálmica interna foi identificado com um padrão levemente dicrótico, ou seja, apresentava dois picos, um mais evidente e afilado e outro

Quadro 1. Valores médios e desvio padrão encontrados para a câmara anterior, espessura da lente, câmara vítrea e o comprimento axial do bulbo ocular do bugio ruivo (Alouatta fusca)

\begin{tabular}{ccccc}
\hline Olho & D1 $(\mathrm{mm})$ & D2 $(\mathrm{mm})$ & D3 $(\mathrm{mm})$ & D4 (mm) \\
\hline Esquerdo & $2,1 \pm 0,38$ & $3,7 \pm 0,23$ & $10,4 \pm 0,78$ & $19,3 \pm 1,64$ \\
Direito & $2,1 \pm 0,35$ & $3,7 \pm 0,30$ & $10,3 \pm 0,74$ & $19,3 \pm 1,33$
\end{tabular}

D1 = Câmara anterior, D2 = espessura da lente, D3 = câmara vítrea, D4 = comprimento axial do bulbo ocular; Número total de bulbos oculares $=19$.

Quadro 2. Comparativo dos valores médios dos grupos machos e fêmeas para obtenção de diferença significativa dos parâmetros biométricos no plano dorsal de ambos os bulbos oculares (direito e esquerdo) de bugios ruivos (Alouatta fusca)

\begin{tabular}{|c|c|c|c|c|c|c|c|c|c|}
\hline \multirow{2}{*}{ Bulbo ocular } & \multirow{2}{*}{ Grupos } & \multicolumn{2}{|c|}{$\mathrm{D} 1(\mathrm{~mm})$} & \multicolumn{2}{|c|}{$\mathrm{D} 2(\mathrm{~mm})$} & \multicolumn{2}{|c|}{ D3 (mm) } & \multicolumn{2}{|c|}{$\mathrm{D} 4(\mathrm{~mm})$} \\
\hline & & GM & GF & GM & GF & GM & GF & GM & GF \\
\hline \multirow[t]{3}{*}{ Direito } & Média & 2,3 & 2,0 & 3,8 & 3,7 & 10,4 & 10,3 & 16,7 & 15,6 \\
\hline & Desvio padrão & 0,4 & 0,64 & 0,16 & 0,43 & 0,57 & 0,98 & 0,42 & 2,76 \\
\hline & t crítico bilateral & \multicolumn{2}{|c|}{2,365} & \multicolumn{2}{|c|}{2,365} & \multicolumn{2}{|c|}{2,365} & \multicolumn{2}{|c|}{2,365} \\
\hline \multirow[t]{2}{*}{ Esquerdo } & Média & 2,1 & 2,1 & 3,8 & 3,6 & 10,4 & 10,3 & 16,7 & 16,3 \\
\hline & Desvio padrão & 0,12 & 0,19 & 0,02 & 0,02 & 0,57 & 0,80 & 0,73 & 0,67 \\
\hline
\end{tabular}

$\overline{\mathrm{GM}}$ = Grupos machos, $\mathrm{GF}$ = grupos fêmeas; Teste $\mathrm{t}$ de Student com nível de significância <0,05; número total de bulbos oculares = 10 esquerdos (5 GM e 5 GF) e 9 direitos (4 GM e 5 GF).

Quadro 3. Comparativo dos valores médios dos grupos machos e fêmeas para obtenção de diferença significativa dos parâmetros biométricos no plano sagital de ambos os bulbos oculares (direito e esquerdo) de bugios ruivos (Alouatta fusca)

\begin{tabular}{|c|c|c|c|c|c|c|c|c|c|}
\hline \multirow{2}{*}{ Bulbo ocular } & \multirow{2}{*}{ Grupos } & \multicolumn{2}{|c|}{$\mathrm{D} 1(\mathrm{~mm})$} & \multicolumn{2}{|c|}{$\mathrm{D} 2(\mathrm{~mm})$} & \multicolumn{2}{|c|}{ D3 (mm) } & \multicolumn{2}{|c|}{$\mathrm{D} 4(\mathrm{~mm})$} \\
\hline & & GM & GF & GM & $\mathrm{GF}$ & GM & $\mathrm{GF}$ & GM & $\mathrm{GF}$ \\
\hline \multirow[t]{3}{*}{ Direito } & Média & 2,2 & 1,8 & 3,7 & 3,7 & 10,3 & 10,2 & 16,5 & 16,4 \\
\hline & Desvio padrão & 0,21 & 0,45 & 0,32 & 0,32 & 0,6 & 0,98 & 0,37 & 1,03 \\
\hline & t crítico bilateral & \multicolumn{2}{|c|}{2,365} & \multicolumn{2}{|c|}{2,365} & \multicolumn{2}{|c|}{2,365} & \multicolumn{2}{|c|}{2,365} \\
\hline \multirow[t]{2}{*}{ Esquerdo } & Média & 2,2 & 2,0 & 3,8 & 3,6 & 10,5 & 10,4 & 16,7 & 16,7 \\
\hline & Desvio padrão & 0,33 & 0,52 & 0,25 & 0,33 & 0,74 & 1,04 & 0,44 & 0,97 \\
\hline
\end{tabular}

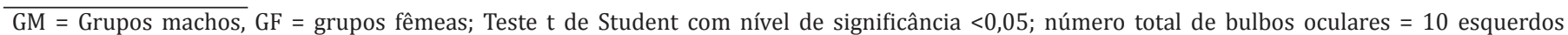
( 5 GM e 5 GF) e 9 direitos (4 GM e 5 GF). 
menos evidente na fase de desaceleração (Fig.4A). Os valores médios e desvios padrão encontrados para os parâmetros de dopplerfluxometria da artéria oftálmica interna foram (Quadro 5): IP de 1,3 $\pm 0,29$ no bulbo ocular esquerdo e $1,4 \pm 0,32$ no bulbo direito; IR de $0,7 \pm 0,07$ no olho esquerdo e $0,7 \pm 0,11$ no direito; VPS de $28,5 \pm 1,89 \mathrm{~cm} / \mathrm{s}$ no bulbo esquerdo e de $24,2 \pm 3,24 \mathrm{~cm} / \mathrm{s}$ no direito; e VDF de $15,8 \pm 7,67 \mathrm{~cm} / \mathrm{s}$ no olho esquerdo e $12,4 \pm 2,66 \mathrm{~cm} / \mathrm{s}$ no direito.

Já o traçado da artéria central da retina apresentou um pico com desaceleração contínua, sem presença de um segundo pico (Fig.4B). Os resultados encontrados para os parâmetros de dopplerfluxometria dessa artéria foram os seguintes (Quadro 6): IP de 0,8 $\pm 0,19$ no olho esquerdo e $0,12 \pm 0,19$ no direito; IR de $0,5 \pm 0,09$ no bulbo ocular esquerdo e $0,5 \pm 0,06$ no direito; VPS de $14,9 \pm 4,39 \mathrm{~cm} / \mathrm{s}$ no bulbo esquerdo e $14,6 \pm 4,17 \mathrm{~cm} / \mathrm{s}$ no direito; e VDF de $10,5 \pm 3,1 \mathrm{~cm} / \mathrm{s}$ no olho esquerdo e $11,0 \pm 3,2 \mathrm{~cm} / \mathrm{s}$ no direito.

Teste comparativos não demonstraram diferença significativa entre os grupos machos e fêmeas em relação aos parâmetros de dopplerfluxometria, obtidos para a artéria oftálmica interna (Quadro 7) e a artéria central da retina (Quadro 8), com valores de t crítico bilateral maiores que o nível de significância $(0,05)$.

Quadro 4. Comparativo dos valores médios dos planos dorsal (PD) e sagital (PS) para obtenção de diferença significativa dos parâmetros biométricos de ambos os bulbos oculares (direito e esquerdo) de bugios ruivos (Alouatta fusca)

\begin{tabular}{|c|c|c|c|c|c|c|c|c|c|}
\hline \multirow{2}{*}{ Bulbo ocular } & \multirow{2}{*}{ Grupos } & \multicolumn{2}{|c|}{$\mathrm{D} 1(\mathrm{~mm})$} & \multicolumn{2}{|c|}{$\mathrm{D} 2(\mathrm{~mm})$} & \multicolumn{2}{|c|}{ D3 (mm) } & \multicolumn{2}{|c|}{$\mathrm{D} 4(\mathrm{~mm})$} \\
\hline & & PD & PD & PD & PS & PD & PS & PD & PS \\
\hline \multirow[t]{3}{*}{ Direito } & Média & 2,1 & 2,0 & 3,8 & 3,7 & 10,4 & 10,3 & 16,1 & 16,5 \\
\hline & Desvio padrão & 0,3 & 0,3 & 0,3 & 0,3 & 0,7 & 0,7 & 2,0 & 0,7 \\
\hline & t crítico bilateral & \multicolumn{2}{|c|}{2,12} & \multicolumn{2}{|c|}{2,12} & \multicolumn{2}{|c|}{2,12} & \multicolumn{2}{|c|}{2,12} \\
\hline \multirow[t]{3}{*}{ Esquerdo } & Média & 2,1 & 2,1 & 3,7 & 3,7 & 10,3 & 10,4 & 21,9 & 16,7 \\
\hline & Desvio padrão & 0,37 & 0,42 & 0,18 & 0,29 & 0,78 & 0,85 & 0,73 & 0,71 \\
\hline & t crítico bilateral & \multicolumn{2}{|c|}{2,101} & \multicolumn{2}{|c|}{2,101} & \multicolumn{2}{|c|}{2,101} & \multicolumn{2}{|c|}{2,101} \\
\hline
\end{tabular}

$\overline{\text { PD }=\text { Plano dorsal, } \mathrm{PS}}=$ plano sagital; Teste t de Student com nível de significância <0,05; número total de bulbos oculares = 10 esquerdos e 9 direitos.

Quadro 5. Média e desvio padrão dos parâmetros de dopplerfluxometria da artéria oftálmica interna do bulbo ocular direito e esquerdo de primatas da espécie Alouatta fusca, incluindo o índice de pulsatilidade, índice de resistividade, velocidade de pico sistólico e velocidade diastólica final

\begin{tabular}{|c|c|c|c|c|}
\hline Olho & IP & IR & VPS $(\mathrm{cm} / \mathrm{s})$ & $\operatorname{VDF}(\mathrm{cm} / \mathrm{s})$ \\
\hline Esquerdo & $1,3 \pm 0,29$ & $0,7 \pm 0,07$ & $28,5 \pm 1,89$ & $15,8 \pm 7,67$ \\
\hline Direito & $1,4 \pm 0,32$ & $0,7 \pm 0,11$ & $24,2 \pm 3,24$ & $12,4 \pm 2,66$ \\
\hline
\end{tabular}

$\overline{\mathrm{IP}}=$ Índice de pusatilidade, $\mathrm{IR}$ = índice de resistividade, VPS = velocidade de pico sistólico, VDF = velocidade diastólica final; número total de bulbos oculares $=20$.
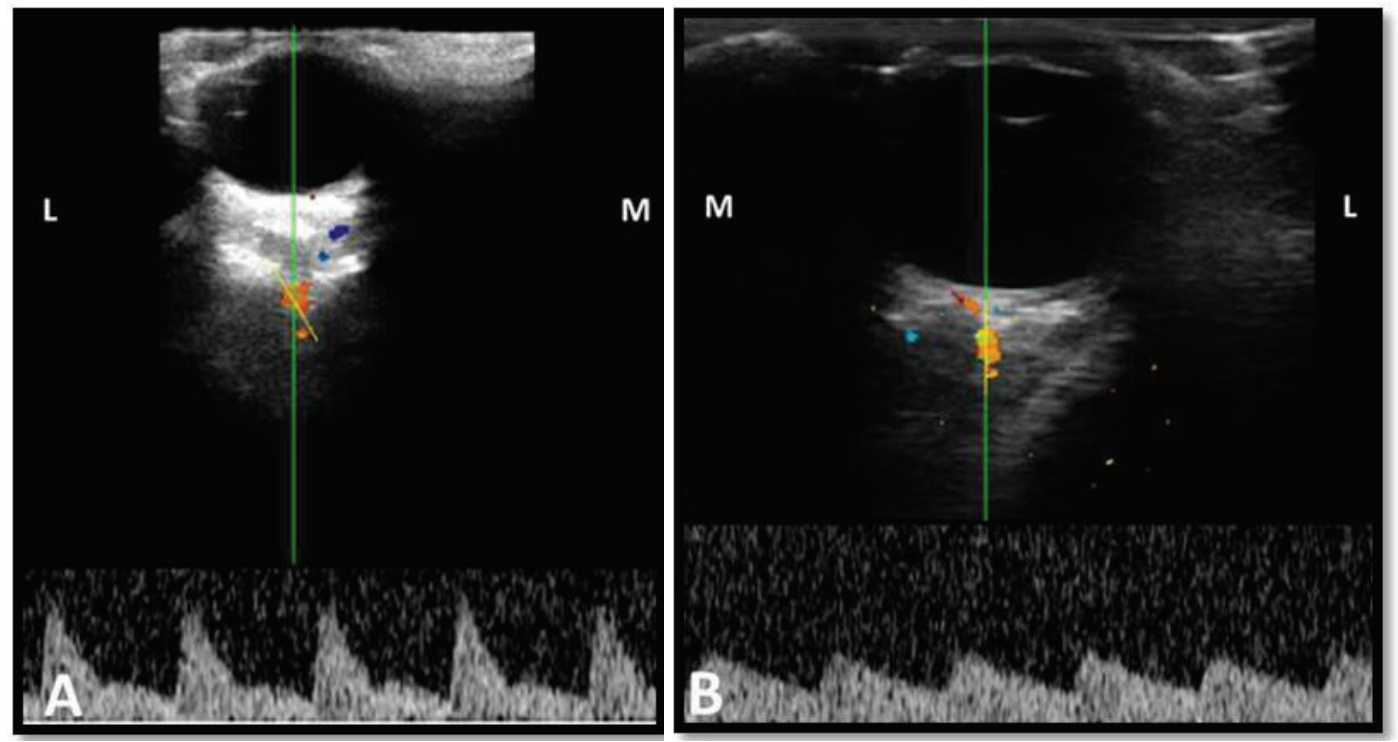

Fig.4. Localização do fluxo e traçado espectral (A) das artérias oftálmica interna e (B) central da retina do bulbo ocular direito, indicando a porção medial (M) e a porção lateral (L) do bulbo ocular. 
Quadro 6. Média e desvio padrão dos parâmetros de dopplerfluxometria da artéria central da retina do bulbo ocular direito e esquerdo de primatas da espécie Alouatta fusca, incluindo o índice de pulsatilidade (IP), índice de resistividade (IR), velocidade de pico sistólico (VPS) e velocidade diastólica final (VDF)

\begin{tabular}{ccccc}
\hline Olho & IP & IR & VPS (cm/s) & VDF (cm/s) \\
\hline Esquerdo & $0,8 \pm 0,19$ & $0,5 \pm 0,09$ & $14,9 \pm 4,39$ & $10,5 \pm 3,1$ \\
Direito & $0,12 \pm 0,19$ & $0,5 \pm 0,06$ & $14,6 \pm 4,17$ & $11,0 \pm 3,2$
\end{tabular}

IP = Índice de pusatilidade, IR = índice de resistividade, VPS = velocidade de pico sistólico, VDF = velocidade diastólica final; número total de bulbos oculares $=18$.

Quadro 7. Comparativo dos valores médios dos grupos machos e fêmeas para obtenção de diferença significativa dos parâmetros de dopplerfluxometria da artéria oftálmica interna, de ambos os bulbos oculares (direito e esquerdo) de bugios ruivos (Alouatta fusca)

\begin{tabular}{|c|c|c|c|c|c|c|c|c|c|}
\hline \multirow{2}{*}{ Bulbo ocular } & \multirow{2}{*}{ Grupos } & \multicolumn{2}{|c|}{ IP } & \multicolumn{2}{|c|}{ IR } & \multicolumn{2}{|c|}{ VPS $(\mathrm{cm} / \mathrm{s})$} & \multicolumn{2}{|c|}{ VDF $(\mathrm{cm} / \mathrm{s})$} \\
\hline & & GM & GF & GM & GF & GM & $\mathrm{GF}$ & GM & GF \\
\hline \multirow[t]{2}{*}{ Direito } & Média & 1,3 & 1,5 & 0,7 & 0,7 & 26,1 & 22,2 & 13,8 & 11,0 \\
\hline & Desvio padrão & 0,10 & 0,12 & 0,005 & 0,02 & 11,6 & 4,56 & 7,88 & 5,03 \\
\hline \multirow[t]{2}{*}{ Esquerdo } & Média & 1,2 & 1,4 & 0,7 & 0,7 & 30,2 & 26,9 & 17,0 & 14,7 \\
\hline & Desvio padrão & 0,23 & 0,37 & 0,07 & 0,08 & 13,73 & 12,59 & 7,92 & 9,00 \\
\hline
\end{tabular}

$\overline{\mathrm{GM}}=$ Grupos machos, GF = grupos fêmeas; Teste $\mathrm{t}$ de Student com nível de significância <0,05; número total de bulbos oculares = 20 (10 GM e 10 GF).

Quadro 8. Comparativo dos valores médios dos grupos machos e fêmeas para obtenção de diferença significativa dos parâmetros de dopplerfluxometria da artéria central da retina, de ambos os bulbos oculares (direito e esquerdo) de bugios ruivos (Alouatta fusca)

\begin{tabular}{|c|c|c|c|c|c|c|c|c|c|}
\hline \multirow{2}{*}{ Bulbo ocular } & \multirow{2}{*}{ Grupos } & \multicolumn{2}{|c|}{ IP } & \multicolumn{2}{|c|}{ IR } & \multicolumn{2}{|c|}{ VPS $(\mathrm{cm} / \mathrm{s})$} & \multicolumn{2}{|c|}{$\operatorname{VDF}(\mathrm{cm} / \mathrm{s})$} \\
\hline & & GM & GF & GM & GF & GM & $\mathrm{GF}$ & GM & $\mathrm{GF}$ \\
\hline \multirow[t]{2}{*}{ Direito } & Média & 0,69 & 0,69 & 0,51 & 0,4 & 16,2 & 13,3 & 12,1 & 10,1 \\
\hline & Desvio padrão & 0,09 & 0,15 & 0,04 & 0,07 & 6,3 & 2,1 & 4,81 & 1,88 \\
\hline \multirow[t]{2}{*}{ Esquerdo } & Média & 0,8 & 0,7 & 0,6 & 0,5 & 18,0 & 12,4 & 12,2 & 9,0 \\
\hline & Desvio padrão & 0,12 & 0,24 & 0,06 & 0,11 & 4,02 & 3,73 & 2,02 & 3,57 \\
\hline
\end{tabular}

$\overline{\mathrm{GM}}=$ Grupos machos, GF $=$ grupos fêmeas; Teste t de Student com nível de significância <0,05; número total de bulbos oculares = 9 esquerdos ( 4 GM e 5 GF) e 9 direitos (4 GM e $5 \mathrm{GF}$ ).

\section{DISCUSSÃO}

A imagem do bulbo ocular é bastante fácil de ser obtida devido às diferenças de impedância das suas estruturas, o que o torna um órgão ideal para o exame ultrassonográfico (Mattoon \& Nyland 2005). A descrição precisa da anatomia do bulbo ocular de primatas não humanos ainda é escassa, porém, os aspectos ultrassonográficos nesta espécie não diferem das principais espécies domésticas descritas por alguns autores, como cães e gatos (Mattoon \& Nyland 2005, Hernández-Guerra et al. 2007). Sendo assim, a obtenção das imagens do bulbo ocular em primatas da espécie Alouatta fusca foi realizada com facilidade, com reconhecimento rápido das estruturas oculares, possivelmente devido à semelhança com as demais espécies.

Para análise da cápsula lenticular em toda sua extensão, foi realizada a varredura completa, pois por muitas vezes, suas porções mais laterais não eram caracterizadas em somente uma imagem. Autores relatam que a não caracterização destas porções da lente ocorre também em cães e gatos, por tratar-se de uma estrutura de superfície curvilínea, em que há saída periférica do eco devido à refração e reflexão do feixe sonoro (Mattoon \& Nyland 2005).

Em diversos momentos, durante a obtenção da imagem das estruturas anecóicas, em especial da câmara vítrea, foram observadas linhas ecogênicas paralelas, representando artefato de reverberação. Este artefato é frequente e tende a desaparecer conforme o ângulo do corte é mudado, não representando prejuízos à realização e interpretação do exame (Mattoon \& Nyland 2005, Hernández-Guerra et al. 2007).

Os pontos de referência utilizados neste estudo para mensuração das diferentes distâncias na biometria ocular dos bugios ruivos foram semelhantes àqueles citados por estudo de Ribeiro et al. (2009) e Ruiz et al. (2015), realizados em caprinos Saanen e jacarés-do-Pantanal, respectivamente; incluindo a profundidade da câmara anterior (D1), a espessura da lente (D2), a profundidade da câmara vítrea (D3) e o comprimento axial do bulbo ocular (D4).

Os valores do comprimento axial do bulbo ocular e a espessura da lente encontrados mostraram-se discretamente menores em relação ao gorila (Liang et al. 2005) e ao ser 
humano (Hoffer 1980), e maiores em comparação ao macaco rhesus (Qiao-Grider et al. 2007). A profundidade da câmara anterior observada nos bugios mostrou-se mais superficial em relação ao gorila (Liang et al. 2005), ao homem (Hoffer 1980) e ao macaco rhesus (Qiao-Grider et al. 2007). Já a mensuração da câmara vítrea apresentou-se mais larga em relação a essa última espécie (Qiao-Grider et al. 2007).

A análise estatística foi realizada com o objetivo de detectar possível diferença dos valores de mensuração entre machos e fêmeas e entre os cortes ultrassonográficos sagital e dorsal. Presumia-se que não houvesse diferença significativa em relação ao gênero, possivelmente devido a pouca alteração de peso entre machos e fêmeas e, em relação aos cortes, por se tratar da mesma estrutura; em oposição ao que ocorre com os macacos rhesus, nos quais os machos costumam ter olhos mais largos que as fêmeas (Qiao-Grider et al. 2007). Após a realização do teste $\mathrm{t}$ de Student, em que o valor de t crítico bilateral foi maior do que 0,05 , em ambas as comparações, pode-se comprovar que não há diferença significativa entre gêneros e cortes ultrassonográficos.

Anatomicamente, as estruturas oculares examinadas pelo modo $\mathrm{B}$ foram muito semelhantes àquelas descritas em estudos humanos e veterinários. 0 bulbo ocular é caracterizado como uma estrutura arredondada bem demarcada, formada pelas estruturas lineares hiperecóicas córnea, cápsula anterior e posterior da lente e complexo retina-coróide-esclera, que delimitam áreas anecóicas centrais - as câmaras anterior e posterior, a lente e a câmara vítrea, numa análise anteroposterior (Gonzalez et al. 2001, Mattoon \& Nyland 2005, Ruiz et al. 2015).

Devido a pouca base de dados sobre a anatomia vascular do bulbo ocular na medicina veterinária, este estudo procurou seguir a orientação anatômica fornecida em trabalhos humanos, no qual foi relatada a anatomia sonográfica do bulbo ocular e das técnicas de obtenção da velocidade de fluxo das artérias e veias retrobulbares com recurso de dopplerfluxometria (Williamson et al. 1995, Williamson \& Harris 1996, Diniz et al. 2004).

Em estudo realizado por Brooks et al. (2007), as mensurações da artéria oftálmica foram obtidas de forma semelhante, medialmente ao nervo óptico em sua porção superficial. A VPS encontrada é menor em relação aos humanos (Williamson \& Harris 1996, Diniz et al. 2004) e ao macaco rhesus (Brooks et al. 2007). Já a VDF demonstrou um intervalo de variação amplo, com valores entremeio aos encontrados em humanos (Williamson \& Harris 1996) e em macacos rhesus (Brooks et al. 2007). 0 índice de resistividade e pulsatilidade da artéria oftálmica observado nos bugios mostraram-se semelhantes ao macaco rhesus e aos homens, respectivamente (Williamson \& Harris 1996, Brooks et al. 2007). Quanto à artéria central da retina, a VPS e VDF mostraram-se maiores em relação aos humanos.

$\mathrm{Na}$ maioria dos animais, a identificação de fluxo foi realizada, porém a obtenção do traçado espectral da artéria central da retina foi dificultosa em dois bulbos oculares de animais diferentes, ambos com idade avançada (10 e 12 anos). Williamson et al. (1995) demonstraram em um estudo realizado com 95 pessoas, que o avançar da idade está negativamente correlacionado a velocidade do fluxo sanguíneo e positivamente envolvido com a resistência do fluxo na artéria central da retina, podendo ser uma explicação para a maior dificuldade encontrada na avaliação ecográfica via dopplerfluxometria.
Da mesma maneira, observou-se que em animais jovens as ondas apresentavam um traçado mais forte do que em animais de idade avançada. Como supracitado, a idade pode ser um dos fatores de influência na velocidade do fluxo orbital em humanos, pelo aumento da pressão arterial, diminuição do lúmen ou enrijecimento da parede de alguns vasos peribulbares (Williamson et al. 1995), contudo não foi possível mensurar a influência da idade sobre os parâmetros de dopplerfluxometria da artéria central da retina nos primatas da espécie Alouatta fusca utilizados no presente estudo.

\section{CONCLUSÕES}

Valores de base para mensuração das estruturas do bulbo ocular da espécie Alouatta fusca foram obtidos através da biometria ocular, mostrando não haver diferença estatística em relação ao gênero dos animais e ao plano de corte ecográfico utilizado.

Foram obtidos aspectos normais do traçado espectral e valores de base para os parâmetros de dopplerfluxometria das artérias oftálmica interna e central da retina, não apresentando diferença significativa entre o sexo dos animais.

Os aspectos ultrassonográficos das estruturas oculares de primatas não humanos da espécie estudada não diferem em relação às principais espécies domésticas, como cães e gatos, e tampouco da espécie humana.

A ultrassonografia é um método de imagem de fácil acesso e execução, desde que realizado por profissionais dotados de conhecimento e treinamento adequados. Tem-se mostrado uma técnica de grande valia para avaliação oftalmológica, sendo um campo aberto para estudo e pesquisa na medicina veterinária.

Os resultados obtidos neste estudo servirão como referência para estudos futuros e para a clínica veterinária de animais silvestres, auxiliando no diagnóstico de afecções como opacificação de córnea, neoplasias, glaucoma e descolamento de retina.

Além disso, em função da semelhança anatômica detectada com o bulbo ocular humano, permite que tal espécie seja utilizada como modelo experimental em pesquisas da medicina humana.

Agradecimentos.- Ao Centro de Medicina e Pesquisa em Animais Silvestres (CEMPAS), Faculdade de Medicina Veterinária e Zootecnia, UNESP/Campus de Botucatu, pelos animais deste estudo.

\section{REFERÊNCIAS}

Auricchio P. 1995. Primatas do Brasil. Terra Brasilis, São Paulo, p.26-28.

Brooks D.E., Komaromy A.M., Kallberg M.E., Miyabashi T., Ollivier F.J. \& Lambrou G.N. 2007. Blood flow velocity response of the ophthalmic artery and anterior optic nerve head capillaries to carbogen gas in the rhesus monkey modelo f optic nerve head ischemia. Vet. Ophthalmol. 10(Suppl.1):20-27. PMid:17973831.

Carvalho C.F., Dupré A.S.A. \& Perez R.B. 2009. Ultrassonografia doppler ocular, p.152-157. In: Carvalho C.F. (Ed), Ultrassonografia Doppler em Pequenos Animais. Roca, São Paulo.

Diniz A.L.D., Moron A.F., Santos M.C. \& Sass N. 2004. Dopplervelocimetria colorida dos vasos orbitais: técnica de exame e anatomia vascular. Radiol. Bras. 37(4):287-290.

Dyce K.M., Sack W.O. \& Wensing C.J.G. 2004. Tratado de Anatomia Veterinária. 3 a ed. Guanabara Koogan, Rio de Janeiro, p.318-322. 
Gonzalez E.M., Rodriguez A. \& Garcia I. 2001. Review of ocular ultrasonography. Vet. Radiol. Ultrasound 42(6):485-495. PMid:11768514.

Hernández-Guerra A.M., Rodilla V. \& López-Murcia M.M. 2007. Ocular biometry in the adult anesthetized ferret (Mustela putorius furo). Vet. Ophthalmol. 10(1):50-52. PMid:17204128.

Hoffer K.J. 1980. Biometry of 7500 cataractous eyes. Am. J. Ophthalmol. 90(3):360-368. PMid:7425052.

Liang D., Alvarado T.P., Oral D., Vargas J.M., Denena M.M. \& McCulley J.P. 2005. Ophthalmic examination of the captive Western Lowland Gorilla (Gorilla gorilla gorilla). J. Zoo Wildl. Med. 36(3):430-433. PMid:17312760.

Marques K.V. 2005. Estudo anatômico do tronco encefálico do macaco Cebus apella. Dissertação de Mestrado, Faculdade de Medicina Veterinária e Zootecnia, Universidade de São Paulo, São Paulo. 8p.

Mattoon J.S. \& Nyland T.G. 2005. Olho, p.315-335. In: Nyland T.G. \& Mattoon J.S. (Eds), Ultrassom Diagnóstico em Pequenos Animais. 2ª ed. W.B. Saunders, São Paulo.

Montiani-Ferreira F., Shaw G., Mattos B.C., Russ H.H. \& Vilani R.G. 2008. Reference for selected ophthalmic diagnostic tests of the capuchin monkey (Cebus apella). Vet. Ophthalmol. 11(3):197-201. PMid:18435663.

Morgan R.V. 1989. Ultrasonography of retrobulbar diseases of the dog and cat. J. Am. Anim. Hosp. Assoc. 25(4):393-399.
Pippi N.L. \& Gonçalves G.F. 2009. Anatomofisiologia ocular, p.1-10. In: Laus J.L. (Ed), Oftalmologia Clínica e Cirúrgica em Cães e Gatos. Roca, São Paulo.

Qiao-Grider Y., Hung L.-F., Kee C., Ramamirtham R. \& Smith 3rd E.L. 2007. Normal ocular development in young rhesus monkey (Macaca mulatta). Vision Research 47(11):1424-1444. PMid:17416396.

Ribeiro A.P., Silva M.L., Rosa J.P., Souza S.F., Teixeira I.A.M.A. \& Laus J.L. 2009 Ultrasonographic and echobiometric findings in the eyes of Saanen goats of different ages. Vet. Ophthalmol. 12(5):313-317. PMid:19751492.

Ruiz T., Campos W.N.S., Peres T.P.S., Gonçalves G.F., Ferraz R.H.S., Néspoli P.E.B., Sousa V.R.F. \& Ribeiro A.P. 2015. Intraocular pressure, ultrasonographic and echobiometric findings of juvenile Yacare caiman (Caiman yacare) eye. Vet. Ophthalmol. 18(Suppl.1):40-45. PMid:24450942.

Samuelson D.A. 2007. Ophtalmic anatomy, p.37-148. In: Gelatt K.N. (Ed.), Veterinary Ophthalmology. 4th ed. Blackwell Publishing, Iowa.

Toni M.C., Meirelles A.E.W.B., Gava F.N., Camacho A.A., Laus J.L. \& Canola J.C. 2010. Rabbit's eye globe sonographic biometry. Vet. Ophthalmol. 13(6):384-386. PMid:21182723.

Williamson T.H. \& Harris A. 1996. Color doppler ultrasound imaging of the eye and orbit. Survey Ophthalmol. 40(4):255-267. PMid:8658337.

Williamson T.H., Lowe G.D. \& Baxter G.M. 1995. The influence of age, systemic blood pressure, smoking and blood viscosity on orbital blood velocities. Brit. J. Ophthalmol. 79(1):17-22. PMid:7880783. 